\title{
The effects of freeze drying and freeze drying additives on the prothrombin time and the international sensitivity index
}

\author{
L Poller, M Keown, S A Shepherd, C R Shiach, S Tabeart
}

\begin{abstract}
Aim-To determine whether freezing, freeze drying protective additives, or freeze drying of plasma samples from patients on coumarin treatment and from normal individuals affects prothrombin times or the international sensitivity index (ISI) calibration.
\end{abstract}

Methods-The effect of the addition of the protective additives singly and combined on the prothrombin time of coumarin samples and normal samples before and after freeze drying was observed using high and low ISI reference thromboplastins. ISI values were also determined.

Results-Freezing caused a prolongation of prothrombin time in the normal plasma samples with both reagents, which was significant with the low ISI human. Prolongation (non-significant) of the prothrombin time in coumarin plasma samples occurred with the human reagent only. Significant prolongation of normal prothrombin time by some of the protective additives before and after freeze drying was observed with both thromboplastins but to a greater extent with the human. Significant prolongation of prothrombin time in coumarin plasma samples was observed, but again was more marked with human thromboplastin. An approximate ISI was determined on the 20 coumarin samples. The only marked ISI change was with the WHO human thromboplastin after freeze drying of plasma, where a decrease from 0.95 to 0.90 was observed, corresponding to a marked prothrombin ratio increase

Conclusions-Freeze drying additives and the freeze drying procedure prolong normal and coumarin prothrombin times, with low ISI thromboplastin. Less marked prolongations occurred with a high ISI rabbit reagent, coumarin samples showing more significant prolongations. Marked ISI change in freeze dried plasma was only recorded with the low ISI ECAA human reagent. Frozen normal plasma samples cannot be used with confidence for ISI calibrations.

(F Clin Pathol 1999;52:744-748)

Keywords: freeze drying; additives; prothrombin time; ISI

Freeze dried plasma as a replacement for fresh plasma is being used on an increasing scale for the international sensitivity index (ISI) calibra- tion of local prothrombin time systems (thromboplastin/coagulometer combinations) or local international normalised ratio (INR) correction..$^{1-4} \mathrm{~A}$ recent collaborative study by the European Concerted Action on Anticoagulation (ECAA) at 14 national control laboratories showed that freeze dried plasma samples, when substituted for fresh coumarin plasma samples, gave a reasonable approximation to thromboplastin calibration slopes obtained with the fresh coumarin samples. Small but measurable differences from fresh plasma calibrations were, however, observed and it was suggested that these may have been caused by the protective additives (Hepes, glycine, sucrose), the freeze drying process, or a combination of these. ${ }^{5}$

In the present study plasma samples from patients stabilised on long term coumarin (warfarin) treatment and from healthy normal subjects were studied in order to identify which if any of the various procedures or protective additives that are normally part of the process of freeze drying plasma cause changes in prothrombin times or ISI, or both.

We studied the effects of freezing and the addition of protective freeze drying additives (an integral step in the freeze drying process) on manual prothrombin time and ISI values obtained with these plasma samples singly and in combination. We also studied the effects of freeze drying on blood samples from the same donor in two laboratories.

\section{Methods}

Blood samples were obtained from long term patients stabilised on coumarin (warfarin) treatment and from healthy normal Manchester University staff volunteers. From each patient and normal subject $20 \mathrm{ml}$ of blood were dispensed into four $5 \mathrm{ml}$ sample tubes containing $3.2 \%$ sodium citrate. An attempt was made to provide a wide and even spread of INR across the INR interval of 1.5 to 4.0 . Donations were obtained from 20 coumarin patients and seven healthy normal subjects, this being the established minimum number of samples required for reliable local ISI calibration with manual technique using an ECAA thromboplastin. ${ }^{67}$ Three additional coumarin plasma samples were collected as a safeguard against duplication of INR values. No values were in fact duplicated but to avoid possible bias all 23 coumarin samples were included in the analysis.

The samples were centrifuged at $2823 \mathrm{~g}$ for 10 minutes at $4^{\circ} \mathrm{C}$. Twelve $0.5 \mathrm{ml}$ plasma 
Table 1 Mean prothrombin times of normal plasma samples $(n=7)$, with range from which mean was derived in parentheses

\begin{tabular}{|c|c|c|c|c|c|c|c|}
\hline & Fresh & Frozen & Hepes & Glycine & Sucrose & All & Freeze dried \\
\hline \multicolumn{8}{|l|}{ (A) ECAA rabbit } \\
\hline Laboratory 1 & $\begin{array}{l}16.96 \\
(16.4 \text { to } 17.4)\end{array}$ & $\begin{array}{l}17.4 \\
(16.4 \text { to } 18.1)\end{array}$ & $\begin{array}{l}17.68 \\
(16.15 \text { to } 18.4)\end{array}$ & $\begin{array}{l}17.88 \\
(16.4 \text { to } 18.5)\end{array}$ & $\begin{array}{l}18.51 \\
\text { (17.7 to } 19.4)\end{array}$ & $\begin{array}{l}18.3 \\
(17.6 \text { to } 19.0)\end{array}$ & $\begin{array}{l}18.54 \\
\text { (18.1 to } 19.5)\end{array}$ \\
\hline Laboratory 2 & $\begin{array}{l}18.4 \\
(17.3 \text { to } 18.6)\end{array}$ & $\begin{array}{l}18.61 \\
(17.5 \text { to } 19.5)\end{array}$ & $\begin{array}{l}18.56 \\
(18.5 \text { to } 19.2)\end{array}$ & $\begin{array}{l}18.64 \\
(17.1 \text { to } 19.6)\end{array}$ & $\begin{array}{l}19.43 \\
(18.0 \text { to } 21.0)\end{array}$ & $\begin{array}{l}18.93 \\
(18.0 \text { to } 20.2)\end{array}$ & $\begin{array}{l}19.11 \\
(17.0 \text { to } 20.2)\end{array}$ \\
\hline Mean & 17.55 & 17.88 & 18.12 & 18.26 & 18.97 & 18.62 & 18.83 \\
\hline $\mathrm{p}$ Value (1) & & $<0.1$ & 0.01 & $<0.01$ & 0.0001 & 0.0005 & 0.001 \\
\hline $\mathrm{p}$ Value (2) & & & & & & & 0.34 \\
\hline \multicolumn{8}{|l|}{ (B) WHO human } \\
\hline Laboratory 1 & $\begin{array}{l}12.31 \\
\text { (11.0 to } 13.45)\end{array}$ & $\begin{array}{l}12.74 \\
\text { (11.7 to } 13.65)\end{array}$ & $\begin{array}{l}12.79 \\
(11.8 \text { to } 13.6)\end{array}$ & $\begin{array}{l}12.99 \\
(12.25 \text { to } 13.8)\end{array}$ & $\begin{array}{l}13.22 \\
(12.0 \text { to } 14.6)\end{array}$ & $\begin{array}{l}13.49 \\
(12.85 \text { to } 14.1)\end{array}$ & $\begin{array}{l}12.91 \\
(11.6 \text { to } 13.6)\end{array}$ \\
\hline Laboratory 2 & $\begin{array}{l}11.94 \\
(11.1 \text { to } 13.0)\end{array}$ & $\begin{array}{l}12.66 \\
(10.9 \text { to } 13.9)\end{array}$ & $\begin{array}{l}12.51 \\
(11.5 \text { to } 13.8)\end{array}$ & $\begin{array}{l}12.87 \\
(11.4 \text { to } 13.7)\end{array}$ & $\begin{array}{l}12.6 \\
\text { (11.8 to } 13.5)\end{array}$ & $\begin{array}{l}13.13 \\
(12.2 \text { to } 13.9)\end{array}$ & $\begin{array}{l}13.64 \\
(12.5 \text { to } 14.8)\end{array}$ \\
\hline Mean & 12.13 & 12.7 & 12.65 & 12.93 & 12.91 & 13.31 & 13.28 \\
\hline p Value (1) & & $<0.01$ & 0.02 & $<0.0001$ & $<0.001$ & $<0.0001$ & 0.0005 \\
\hline $\mathrm{p}$ Value (2) & & & & & & & 0.83 \\
\hline
\end{tabular}

p Value (1), comparison with fresh plasmas; $p$ value (2), comparison of all additives with freeze drying (5\% significance).

WHO, World Health Organisation.

aliquots were dispensed into individual containers, two being retained for "fresh" testing and the remainder snap frozen at $-40^{\circ} \mathrm{C}$. Tests on the fresh samples were performed as soon as possible, the tests being completed in both laboratories within two hours of collection. The remainder of the sample of plasma from each patient and normal subject was also snap frozen at $-40^{\circ} \mathrm{C}$ for freeze drying. Fresh samples could not have been used with confidence to study the effects of the protective additives and freeze drying because of the effects of the varying time intervals between sample collection and testing.

After freezing at $-40^{\circ} \mathrm{C}$ for seven days, individual aliquots were thawed rapidly and manual prothrombin time estimations were performed at both laboratories on the samples without any additive. We observed the effects on prothrombin time of the following freeze drying protective additives dissolved in distilled water, added singly and combined to an aliquot after thawing: glycine $2.18 \mathrm{~g} / \mathrm{dl}$, Hepes 0.26 $\mathrm{g} / \mathrm{dl}$, and sucrose $2.18 \mathrm{~g} / \mathrm{dl}$. Tests were completed on an aliquot before the next was thawed and the individual or combined additives were added. The protective additives added in this study were routinely used in freeze drying of the ECAA calibrant plasmas.

Prothrombin times were measured in parallel by the same two experienced personnel in the two different laboratories using the manual technique. Tests were performed in duplicate within 30 minutes of removal of an aliquot from the freezer.

After the seven days of storage at $-40^{\circ} \mathrm{C}$ the frozen residual sample of each plasma (normal and coumarin) was thawed and the prothrombin time tested. Owing to the restricted volume of the plasmas, there was only sufficient volume for the study of the combined additives after freeze drying.

The effects of the various additives and freeze drying procedures were observed on raw prothrombin time results, prothrombin ratios, and ISI values.

The ISI values of the aliquots were determined with the two WHO reference thromboplastins, ECAA human (ISI 0.95, SE 0.0078) and ECAA rabbit (ISI 1.67, SE 0.0322). ${ }^{8}$ The human reagent was used as the reference in all the ISI calibrations and the ECAA rabbit calibrations were based on the cross species calibration against the human reference preparation. The ISI of 0.95 for the human reagent was obtained from a previous multicentre calibration. ${ }^{8}$ In the same multicentre calibration a cross species (human $v$ rabbit reagent) was performed for the ECAA rabbit reference reagent, giving an ISI of 1.66 as opposed to 1.67 with the same species calibration.

In the present study a conventional ISI calibration analysis was performed according to the revised WHO protocol with exclusion

Table 2 Mean prothrombin times of coumarin plasma samples, with range from which mean was derived in parentheses

\begin{tabular}{|c|c|c|c|c|c|c|c|}
\hline & Fresh & Frozen & Hepes & Glycine & Sucrose & All & Freeze dried \\
\hline \multicolumn{8}{|l|}{ (A) ECAA rabbit } \\
\hline Laboratory 1 & $\begin{array}{l}28.86 \\
(18.95 \text { to } 39.25)\end{array}$ & $\begin{array}{l}28.53 \\
(18.25 \text { to } 36.1)\end{array}$ & $\begin{array}{l}29.79 \\
(20.4 \text { to } 39.0)\end{array}$ & $\begin{array}{l}30.05 \\
(19.2 \text { to } 39.5)\end{array}$ & $\begin{array}{l}30.53 \\
(19.8 \text { to } 39.5)\end{array}$ & $\begin{array}{l}30.23 \\
(19.3 \text { to } 39.1)\end{array}$ & $\begin{array}{l}30.94 \\
(21.0 \text { to } 39.0)\end{array}$ \\
\hline Laboratory 2 & $\begin{array}{l}30.78 \\
(21.0 \text { to } 40.4)\end{array}$ & $\begin{array}{l}30.9 \\
(21.0 \text { to } 43.1)\end{array}$ & $\begin{array}{l}31.99 \\
(22.2 \text { to } 40.6)\end{array}$ & $\begin{array}{l}31.22 \\
(20.8 \text { to } 41.7)\end{array}$ & $\begin{array}{l}32.22 \\
(21.5 \text { to } 43.2)\end{array}$ & $\begin{array}{l}31.31 \\
(21.4 \text { to } 44.1)\end{array}$ & $\begin{array}{l}31.99 \\
(21.3 \text { to } 43.9)\end{array}$ \\
\hline Mean & 29.82 & 29.72 & 30.89 & 30.64 & 31.38 & 30.77 & 31.47 \\
\hline $\mathrm{p}$ Value (1) & & 0.7 & 0.16 & 0.0001 & $<0.0001$ & $<0.0001$ & $<0.0001$ \\
\hline $\mathrm{p}$ Value (2) & & & & & & & 0.009 \\
\hline \multicolumn{8}{|l|}{ (B) WHO human } \\
\hline Laboratory 1 & $\begin{array}{l}30.7 \\
(14.8 \text { to } 48.0)\end{array}$ & $\begin{array}{l}31.76 \\
(15.35 \text { to } 52.0)\end{array}$ & $\begin{array}{l}31.94 \\
(14.6 \text { to } 49.65)\end{array}$ & $\begin{array}{l}33.49 \\
(14.9 \text { to } 53.0)\end{array}$ & $\begin{array}{l}32.0 \\
(15.2 \text { to } 49.2)\end{array}$ & $\begin{array}{l}33.02 \\
(15.3 \text { to } 54.0)\end{array}$ & $\begin{array}{l}35.36 \\
(16.7 \text { to } 53.95)\end{array}$ \\
\hline Laboratory 2 & $\begin{array}{l}28.8 \\
(15.3 \text { to } 42.3)\end{array}$ & $\begin{array}{l}29.57 \\
(15.1 \text { to } 47.1)\end{array}$ & $\begin{array}{l}29.96 \\
(15.5 \text { to } 46.1)\end{array}$ & $\begin{array}{l}30.19 \\
(15.5 \text { to } 48.2)\end{array}$ & $\begin{array}{l}29.64 \\
(14.4 \text { to } 47.0)\end{array}$ & $\begin{array}{l}30.66 \\
(15.0 \text { to } 51.2)\end{array}$ & $\begin{array}{l}32.73 \\
(16.3 \text { to } 54.6)\end{array}$ \\
\hline Mean & 29.75 & 30.67 & 30.95 & 31.84 & 30.82 & 31.84 & 34.05 \\
\hline $\mathrm{p}$ Value (1) & & 0.006 & $<0.0001$ & $<0.0001$ & $<0.001$ & $<0.0001$ & $<0.00001$ \\
\hline p Value (2) & & & & & & & 0.0002 \\
\hline
\end{tabular}

p Value (1), comparison with fresh samples; $p$ value (2), comparison of all additives with freeze drying (5\% significance).

WHO, World Health Organisation. 
Table 3 Mean prothrombin time ratios (PR): coumarin plasma samples to normal plasma samples

\begin{tabular}{|c|c|c|c|c|c|c|c|}
\hline & Fresh & Frozen & Hepes & Glycine & Sucrose & All & Freeze dried \\
\hline \multicolumn{8}{|c|}{ (A) ECAA rabbit } \\
\hline PR & 1.69 & 1.66 & 1.7 & 1.67 & 1.65 & 1.65 & 1.67 \\
\hline $\mathrm{p}$ Value (1) & & $<0.01$ & 0.70 & 0.11 & $<0.01$ & $<0.001$ & 0.11 \\
\hline $\mathrm{p}$ Value (2) & & & & & & & $<0.1$ \\
\hline \multicolumn{8}{|c|}{ (B) WHO human } \\
\hline PR & 2.45 & 2.41 & 2.45 & 2.46 & 2.38 & 2.39 & 2.56 \\
\hline $\mathrm{p}$ Value (1) & & 0.34 & 0.38 & 0.39 & 0.029 & 0.137 & 0.0005 \\
\hline $\mathrm{p}$ Value (2) & & & & & & & $<0.0001$ \\
\hline
\end{tabular}

$\mathrm{p}$ Value (1), comparison with fresh plasmas; $\mathrm{p}$ value (2), comparison of all additives with freeze drying ( $5 \%$ significance).

WHO, World Health Organisation.

limits of 3 SD from the calibration line (WHO Expert Committee on Biological Standardisation, $1999^{9}$ ).

The mean prothrombin times of the normal and coumarin samples, with additives singly and combined, were compared with the fresh plasma sample means in an attempt to assess deviations between the various procedures. Samples with combined protective additives were also compared with freeze dried plasma samples. Paired $t$ tests were used to test for significant mean differences. Prothrombin time ratios from coumarin and normal plasma samples were also tested for significance using paired $t$ tests.

\section{Results}

PROTHROMBIN TIME OF NORMAL PLASMA

SAMPLES

The mean prothrombin time of the two laboratories, overall mean prothrombin time, and $\mathrm{p}$ values of differences between the normal plasma results are given in table 1 , together with the normal ranges for the different formulations at the two laboratories. This shows the overall spread of results and the differences between the two laboratories and between the various additives and procedures.

Although there were differences between the two laboratories in absolute prothrombin time results, the overall effect of the additives and freeze drying appeared to be consistent between them.

With the human reference thromboplastin, the normal samples showed a significant prolongation after freezing alone at $-40^{\circ} \mathrm{C}$ for seven days, but with the rabbit reagent the prolongation was not significant.

Table 4 ISI calibrations and coefficient of variation of the slope

\begin{tabular}{llllllll}
\hline & Fresh & Frozen & Hepes & Glycine & Sucrose & All & Freeze dried \\
\hline (A) ECAA rabbit & & & & & & & \\
Laboratory 1 ISI & 1.63 & 1.62 & 1.62 & 1.61 & 1.67 & 1.62 & 1.64 \\
CV & 3.21 & 3.63 & 4.37 & 3.84 & 4.00 & 4.03 & 3.98 \\
Laboratory 2 ISI & 1.55 & 1.59 & 1.55 & 1.59 & 1.60 & 1.62 & 1.56 \\
CV & 3.19 & 2.92 & 2.79 & 3.03 & 3.37 & 3.87 & 4.31 \\
Mean ISI & 1.59 & 1.61 & 1.58 & 1.6 & 1.63 & 1.62 & 1.6 \\
CV & 3.2 & 3.27 & 3.58 & 3.43 & 3.67 & 3.95 & 4.14 \\
& & & & & & & \\
(B) WHO human & & 0.96 & 0.94 & 0.91 & 0.97 & 0.97 & 0.88 \\
Laboratory 1 ISI & & 2.21 & 2.15 & 2.07 & 2.14 & 2.39 & 2.88 \\
CV & 0.98 & 0.96 & 0.98 & 0.97 & 0.97 & 0.93 \\
Laboratory 2 ISI & & 2.02 & 1.59 & 2.07 & 2.37 & 2.27 & 2.70 \\
CV & & 0.97 & 0.95 & 0.94 & 0.97 & 0.97 & 0.9 \\
Mean ISI & 2.04 & 1.87 & 2.07 & 2.25 & 2.33 & 2.49 \\
CV & & & & & & & \\
\hline
\end{tabular}

$\mathrm{CV}$, coefficient of variation; WHO, World Health Organisation.
All the other additives, with the exception of Hepes buffer with the rabbit reagent singly and in combination, gave significant prolongation of prothrombin time with both reference reagents. The effects on the WHO human reference reagent were, however, more marked throughout.

Freeze drying in the presence of all the additives produced no further significant prolongation effect of the normal prothrombin time value with either reagent.

\section{PROTHROMBIN TIME OF COUMARIN PLASMA} SAMPLES

The mean prothrombin time of the two laboratories, overall mean prothrombin time, $p$ values, and the individual range from which the coumarin plasma results were derived are given in table 2 .

With the coumarin samples, freezing at $-40^{\circ} \mathrm{C}$ for seven days did not prolong the prothrombin time with the ECAA rabbit reagent but there was a significant prolongation with the low ISI WHO human reagent $(\mathrm{p}=0.006)$. Likewise Hepes buffer did not cause a significant prolongation with ECAA rabbit ( $p>0.05$ ) but a highly significant prolongation with the WHO human $(\mathrm{p}<0.001)$.

The other additives, singly and combined, caused prolongation with both reagents and this was especially marked with the human reagent. The most significant and marked prolongation $(p<0.0001)$ was observed after freeze drying with the WHO human.

A further highly significant prolongation was observed after freeze drying with all additives. This was, however, greatest with the low ISI human thromboplastin $(\mathrm{p}<0.0002)$.

EFFECT ON PROTHROMBIN TIME RATIO

As prothrombin time ratios are an essential component in INR derivation $\left(\mathrm{INR}=\mathrm{PR}^{\mathrm{ISI}}\right)$ it seemed important to include the effects of the freeze drying additives on prothrombin ratios. These are shown in table 3 . With the rabbit thromboplastin significant change was only observed with the combined additives $(\mathrm{p}<0.001)$. A non-significant increase in prothrombin ratio was recorded after freeze drying. With the human reagent, however, increase in the prothrombin ratio was not significant for any of the procedures except freeze drying $(p<0.0005)$. The difference in all additives before and after freeze drying was also highly significant $(\mathrm{p}<0.0001)$.

\section{EFFECTS ON ISI}

Effects on the prothrombin time of the normal and coumarin samples are not necessarily reflected in the ISI. An approximate ISI was determined for each plasma formulation based on the 20 coumarin plasma samples and seven normal samples. With both reagents a lowering of ISI was observed with all additives after freeze drying, this being most marked with the WHO human. There was a marked lowering to 0.90 after freeze drying (established value $=0.95)$ from the ISI results with the additives singly and combined (table 4 ). The precision of the calibrations (coefficient of 
variation (CV) of the calibration slope) was good with the human reference reagent despite the small numbers of calibration plasma samples (27). At both centres the CV of the slope with all formulations using the low ISI human recombinant reagent was less than 3\% (the WHO precision limit).

The higher ISI rabbit reagent showed negligible change with additives, singly, combined, and after freeze drying. Sucrose gave the most marked change. With this reagent, however, the precision was not as good at the two centres. With this reduced calibration, all formulations gave ISI values with a $\mathrm{CV}$ of the calibration slope greater than $3 \%$, but in all cases this is within the original WHO precision limit of $5 \%$.

\section{Discussion}

In this study we attempted to identify changes caused by freezing and the protective additives involved in freeze drying as well as the freeze drying process itself on prothrombin time and ISI.

Freeze dried plasma samples certified with assigned (certified) manual prothrombin time values are now increasingly being used for ISI calibration of local prothrombin time systems. This is because the conventional WHO ISI calibration is very demanding and places a considerable burden on clinical laboratories and thromboplastin manufacturers to collect the required number of fresh normal and patient samples to be tested in parallel with a thromboplastin reference preparation by the manual prothrombin time technique. The ECAA has developed a set of freeze dried plasma samples for local ISI calibration which have been shown to give acceptable thromboplastin calibrations at national control laboratories and in participant centres in field studies. $^{10}$ Measurable differences from the conventional fresh calibration slopes at the same laboratories were, however, identified in these ECAA studies ${ }^{5}$ although they were well within limits of clinical acceptability. ${ }^{9}$ It was stated in the ECAA report that the origin of these measurable differences from fresh plasma calibrations might be the protective additives in the freeze drying process, the process itself, or a combination of these. ${ }^{4}$

In the present study we monitored the effect on prothrombin time, prothrombin time ratio, and the ISI by observing results of the addition of the individual additives singly and combined to donor plasma samples before freeze drying and of combined additives after freeze drying. There was a consistent pattern of results at the two centres. A prolongation of the prothrombin time on normal and coumarin samples by freezing alone was observed with the low ISI human reagent. With the higher ISI ECAA rabbit reagent no such prolongation was observed for the normal samples, but for the coumarin samples a non-significant shortening was recorded. These changes appear worthy of note as it has previously been suggested that frozen samples can be substituted for fresh coumarins in ISI calibrations. Our results suggest that with a low ISI thromboplastin this could introduce a degree of error. With the higher ISI rabbit reagent, there was also a slight but statistically significant prolongation of prothrombin time in the normal samples after the addition of sucrose or additives combined and after freeze drying.

Prolongation of the prothrombin time after freeze drying of normal plasma samples has been reported previously by Poller et al, ${ }^{11}$ van den Besselaar ${ }^{4}$, and in an ECAA study where the same thromboplastins as in this report were included. ${ }^{12}$ The present results are in agreement with the latter study at 143 centres, which returned results with a reference thromboplastin on the seven ECAA normal freeze dried plasma samples. These were compared with the local mean normal thromboplastin time (MNPT) of fresh plasma samples. Good agreement was obtained between the fresh plasma MNPT with local rabbit reagents but with the human type reagents the seven freeze dried normal plasma samples gave a longer MNPT than the fresh plasma MNPT.

In the present study, we observed highly significant prolongations of prothrombin time with the rabbit preparation and the coumarin samples with all additives except Hepes buffer. Significant prolongation was also observed after freeze drying. With the low ISI human reagent, all procedures including freezing caused prolongation in the normal samples. With the coumarin samples there was an even more significant prolongation following freezing, with the additives singly and combined and after freeze drying.

Our finding of a higher coefficient of variation of the calibration slope with the higher ISI rabbit reagent confirms data from an ECAA study of the minimum requirements of fresh plasmas for reliable ISI calibrations. ${ }^{7}$ This shows that a larger number of fresh coumarin plasma samples was required to conform with the WHO recommendation of a 3\% coefficient of variation for the calibration slope when using the high ISI ECCA rabbit reference reagent than with the low ISI human reagent.

With neither reference reagent was there a significant further prolongation of the prothrombin time after freeze drying of the normal samples, but with the coumarins a further highly significant prolongation was recorded after freeze drying. The most marked changes were observed with the low ISI human reagent. With this reagent there was an increase in the prothrombin ratio and a marked decrease in the ISI. For some procedures and protective additives significant changes were observed with the rabbit thromboplastin, but no change was recorded after freeze drying.

It is of interest that freezing alone was observed to have a slight effect on the ISI. In the case of the WHO human, there was a measurable disproportionate prolongation of the normal prothrombin time compared with the coumarin values at both centres, which disturbed the prothrombin ratio and resultant ISI. Although the difference in ISI terms was not great, this study shows that frozen plasma samples, particularly those from normal individuals, should not be regarded as identical to 
freshly collected samples for the purposes of ISI calibration.

This study was supported by grant number BMH1-CT94-1349 of the Biomedical Programme of the European Commission.

1 Clarke K, Taberner DA, Thomson JM, et al. Assessment of value of calibrated lyophilised plasmas to determine intervatue of calibrated lyophilised plasmas to determine inter1992;45:58-60.

2 Houbouyan LL, Goguel AF. Procedure of reference calibrated plasmas for prothrombin time standardisation [abstract]. XIVth Congress of the International Society on Thrombosis and Hemostasis, New York. Thromb Haemost 1993;69:663

3 Poller L, Triplett DA, Hirsh J, et al. The value of plasma calibrants in correcting coagulometer effects on international normalized ratios - an international multicenter study. Am F Clin Pathol 1995;103:358-65.

4 van den Besselaar AMHP. Comparison of lyophilised plasmas with fresh plasmas for calibration of thromboplastin reagents in oral anticoagulant control. $\mathrm{Br} f \mathrm{Haemato}$ 1996;93:437-44.

5 Poller L, van den Besselaar AMHP, Jespersen J, et al. European Concerted Action on Anticoagulation. A comparison of artificially depleted, lyophilised coumarin and fresh coumarin plasmas in thromboplastin calibration. Br F Haematol 1998;101:462-7.
6 Poller L, Barrowcliffe TW, van den Besselaar AMHP, et al. European Concerted Action on Anticoagulation. MiniEuropean Concerted Action on Anticoagulation. Minimum lyophilised plasma requirement for

7 Poller L, van den Besselaar AMHP, Jespersen J, et al. European Concerted Action on Anticoagulation. The effects of sample size on fresh plasma thromboplastin ISI determination. Br F Haematol 1999;105:655-63.

8 Poller L, Barrowcliffe TW, van den Besselaar AMHP, et al. Prepared on behalf of the ECAA by the above Steering Committee. European Concerted Action on Anticoagulation (ECAA) - the multicentre calibration of rabbit and human ECAA reference thromboplastins. Thromb Haemost 1996;76:977-82.

9 WHO Expert Committee on Biological Standardisation 1999. Requirements for thromboplastin and plasmas used to control oral anticoagulant therapy. Geneva: WHO (in press).

10 Poller L, van den Besselaar AMHP, Jespersen J, et al. European Concerted Action on Anticoagulation. Field studies of coagulometer effects on the ISI of ECAA thromboplastins. Thromb Haemost 1998;80:615-23.

11 Poller L, Houghton D, Carroll J. Reliability of the mean normal prothrombin time of fresh plasmas and of the normal value from a lyophilised "normal" plasma in prothrombin ratio determination. Br f Haematol 1994;88:866-73.

12 Poller L, Barrowcliffe TW, van den Besselaar AMHP, et al. European Concerted Action on Anticoagulation (ECAA). Evaluation of a set of lyophilised normal plasmas to establish the normal prothrombin time for coagulometer systems. Thromb Haemost 1998;79:122-8. 